\title{
Unsupervised system for Lexical Disambiguation of Arabic Language using a vote procedure
}

\author{
Merhbene Laroussi \\ Faculty of Sciences of Monastir \\ LATICE Laboratory \\ University of Monastir, Tunisia \\ Aroussi_merhben@hotmail.com
}

\author{
Anis Zouaghi \\ ISSAT Sousse \\ LATICE Laboratory \\ University of Sousse, Tunisia \\ Anis.zouaghi@gmail.com
}

\author{
Mounir Zrigui \\ Faculty of Sciences of Monastir \\ LATICE Laboratory \\ University of Monastir, Tunisia \\ Mounir.Zrigui@fsm.rnu.tn
}

\begin{abstract}
In this paper we propose an unsupervised method for Arabic word sense disambiguation. Using the corpus and the glosses of the ambiguous word, we define a method to generate automatically the context of use for each sense. Since that, we define a similarity measure based on collocation measures to find the most nearest context of use to the sentence containing the ambiguous word. The similarity measure may give more than one sense, for that we define a novel supervised approach called vote procedure. Our work was compared with other related works. We obtained a better rate of disambiguation in the average of $79 \%$.
\end{abstract}

Keywords-Glosses, Stemming, string-matching, Contexts of use, similarity measure, vote procedure.

\section{INTRODUCTION}

The task of identifying the meaning of a word is called word sense disambiguation (WSD), which is one of the oldest problems in natural language processing (NLP) [1]. The benefits of WSD were exploited by many NLP applications such as machine translation, information retrieval, grammatical analysis, speech processing as well as text processing [1].

In this work, we are interested in determining the meaning of Arabic ambiguous words. First, we apply a weighting method, in order to find the stop words in the sentence containing the ambiguous word. After several tests during the experimental study, we established a list of stop words which will be eliminated from the original sentence.

Then, we use the dictionary and a corpus as a resource for the automatic extraction of contexts of use generated for each sense of the ambiguous word. We apply the stemming [2] for the words contained in the glosses of the ambiguous word, then we use the approximate string-matching algorithm [3] to be able to extract the contexts of uses from the corpus used.

Finally, we define a new similarity measure to find the nearest context of use to the original sentence. Some predefined collocation measures were used by the defined similarity measure. In the case where the similarity measures give more than one sense, we define a vote procedure that chooses the correct sense between those proposed.

This paper is structured as follows. We describe in section two some related works. After that, in section three, we give a detailed account of the proposed method for disambiguation of
Arabic words. Since that in section four, we present the results given by our work and the compared works.

\section{RELATED WORKS}

Most of the works related to the WSD were applied to the English; they are classified using the source of knowledge. In fact there are some works applied to Arabic that are described and detailed in what follows.

\section{A. Knowledge Based Methods}

They were introduced in 1970, based on the dictionary, thesaurus and lexicon. Some of them like the Lesk algorithm tested the adequate definitions given by the electronic dictionary.

We can cite the work of Guiassa [4] that is based on a dictionary of use. The senses of words are represented as a conceptual vector, since that the Lesk algorithm is used to count the intersections between the sense candidate $\mathrm{D}(\mathrm{Sj})$ and the words contained in the same context of the ambiguous word.

Also the variants of the Lesk algorithm were evaluated to disambiguate Arabic words [5]. In the first experiment, the original Lesk algorithm was applied using the dictionary as a resource. As a second experience, the Lesk algorithm was modified using Arabic Wordnet and some similarity measures to determine the relatedness between two concepts in Arabic Wordnet. These variants affiliate a score for the most relevant sense of the ambiguous word using two different resources.

\section{B. Corpus Based Methods}

Since the evolution of the statistic methods based on the large text corpus, two principal methods appear.

1) Unsupervised methods. These methods use a nonannotated corpus. The contexts are represented by highdimensional spaces defined by word co-occurrences.

We can cite the Combination of information retrieval methods with Lesk algorithm for Arabic WSD [6], where three information retrieval measures and the latent semantic analysis were applied, to measure the similarity between the context of use for each sense of the ambiguous word and the original sentence. These measures were combined with the Lesk algorithm to achieve an accuracy rate of $73 \%$. 
2) Supervised Methods. They use an annotated training corpus inducing the appropriate classification models.

We can cite the SALAAM system [7] which exploits translational correspondences between words in a parallel Arabic English corpus to annotate Arabic text using English WordNet taxonomy. The idea is that the translated words are grouped into clusters, after that, WordNet is used to identify the appropriate senses in those clusters and a sense criterion is applied to choose the appropriate sense. The chosen senses tags for the words in the cluster are propagated back to their respective contexts in the parallel text.

Also the Naïve Bayes Classifier for AWSD [8] uses a training corpus where each use of ambiguous words is tagged with its correct sense. A Bayes classifier applies the Bayes decision rule to choose a class. This step is preceded by some pre-processing steps (remove all stop words and getting stems from the original sentence containing the word to be disambiguated). A comparison between the cited works and the proposed method will be detailed in the experimental results.

\section{PROPOSED METHOD}

Using the glosses of the ambiguous word and the corpus, we generate the contexts of use for each sense of the ambiguous word. The idea consists on combining the algorithm of stemming [2] to extract the roots, and the algorithm of approximate string matching [3] to find occurrences of the Stems. Stems and their occurrences are saved in the knowledge base. The texts containing these occurrences with the ambiguous word represent the contexts of use. Subsequently, we eliminate stop words from the original sentence, using the tf.idf measure.

The second step of the proposed method is to measure the similarity between the different contexts of use generated, using three collocation measures. Using a Vote procedure we rank these measures. The context proposed by the most ranked collocation measure will represent the most probable sense of the ambiguous word. In what follows we describe with more details each step cited above.

\section{A. Construction of the Context of use: Motivation and implementation}

To maximize the probability of finding the context for each sense, we proposed as a solution to generate the occurrences of the most significant words. The figure 1 below details the architecture of the proposed method for the construction of the context of use.

The extraction of the most significant words is an important task for this work, for that we use the metric of tf.idf to eliminate the non-informative words (in English stop words). Each stop word will be saved in our database, until now we have a predefined list of 30.000 stop words.

Given that the Arabic word has several derivations of the form, we used an algorithm to extract the root and then an algorithm for matching words to find occurrences of this root.

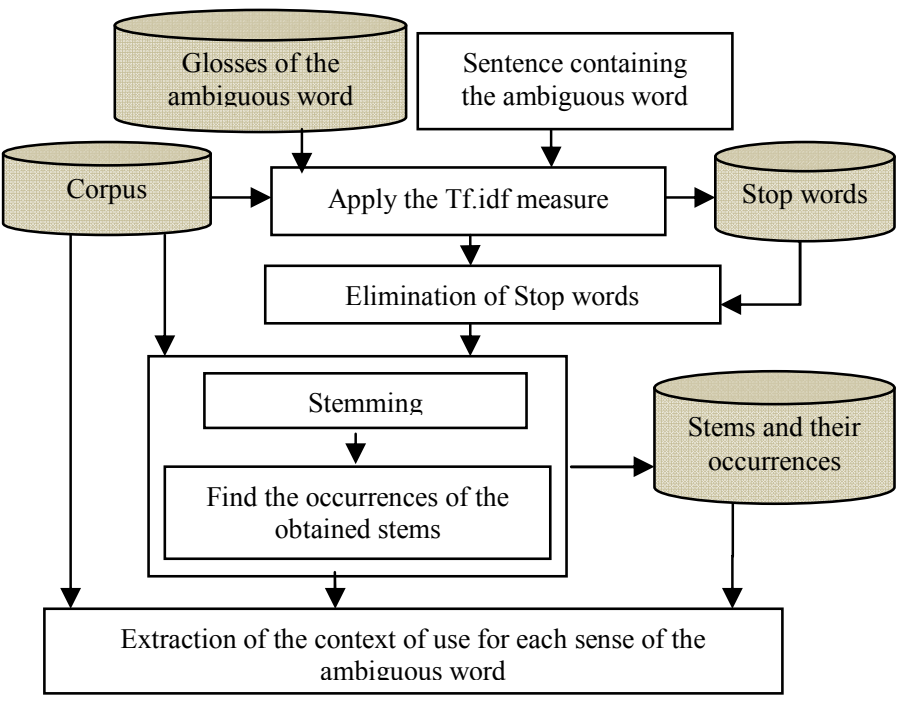

Figure 1. Architecture defined for the automatic extraction of the contexts of use.

Obtaining instances of a root consists on adding a suffix to the beginning of a word or a prefix in the end. In what follows, we detail the steps of the proposed method.

1) Elimination of Stop words: The stop words or non informative words will be eliminated in a first time from the glosses of the ambiguous words, and in a second time from the original sentence (containing the word to be disambiguated). In this work, we use the metric of tf idf [9] that was used in many natural language processing works. This metric is obtained by multiplying the two measures tf and idf :

$$
\mathrm{tf}_{-} \mathrm{idf}_{\mathrm{i}, \mathrm{j}}=\mathrm{tf}_{\mathrm{i}, \mathrm{j}} \times \mathrm{idf}_{\mathrm{i}}
$$

The frequency of a word $\left(\mathrm{tf}_{\mathrm{i}, \mathrm{j}}\right)$ is the number of occurrences af a word in a given document. Let the document $d_{j}$ and the word $t_{i}$, the frequency of $t_{i}$ in $d_{j}$ is measured as follows:

$$
\mathrm{tf}_{\mathrm{i}, \mathrm{j}}=n_{i, j} / \sum_{k} n_{\mathrm{k}, \mathrm{j}}
$$

Where $n_{i, j}$ is the number of occurrences of the word $w_{i}$ in the document $d_{j}$. The denominator is the number of occurrences of all terms in the document $d_{j}$. The inverse document frequency gives the importance of a word in the corpus. It consists on measuring the logarithm of the inverse of the number of documents in the corpus that contain the term:

$$
\operatorname{idf}_{\mathrm{i}}=\log \frac{|D|}{|\{d j: t i \epsilon d j\}|}
$$

Where | D | is the total number of documents in the corpus $\left|\left\{\mathrm{d}_{\mathrm{j}}: \mathrm{t}_{\mathrm{i}} \in \mathrm{d}_{\mathrm{j}}\right\}\right|$, the number of documents where the term $\mathrm{t}_{\mathrm{i}}$ appears.

This weighting method allows us to generate the stop words that do not have an influence on the ambiguous word.

2) Stemming: Each Arabic word is based on three consonants or more rarely four or two letters. These three consonants are the root of the Arabic word, they are the most important consonants used to be compared with other 
consonants used for the derivation of the word (added to the right or left of the root).

In this work, we use the Khoja stemmer that removes the longest suffix and the longest prefix. It then combines the remaining words with verbal and nominal patterns, to extract the root [2].

3) Approximate String Matching: Unlike English, Arabic has a rich derivational system and its one of the characteristics that make it ambiguous.

The Arabic words are based on roots, generally trilateral. We use the algorithm of approximate string matching, to find the possible occurrences of a stem obtained using the khoja stemmer.

This algorithm take so much time in the execution process, for that we decide to generate a database that contains all the derived words from the same root. This algorithm will be applied only for the roots that we don't find in our database.

this algorithm is based on two steps [3]. The first step consists on filling the matrix of the two words to be compared. After that, we use the second step of back-tracking, to find the shortest common subsequence.

The words contained this common subsequence (Stem obtained previously) will be considered as the occurrences of the stem. We use the corpus described in the experimental results to extract the texts containing the words of the glosses and their occurrences. These texts represent the contexts of use.

\section{B. Score measure: Motivation and Implementation}

We propose a new measure that determines the degree of similarity between the sentence containing the ambiguous word and the context of use for a given sense of the ambiguous word. These contexts contain semantically similar words as they represent an extension of glosses that define the ambiguous word. This will enable us to increase the probability of finding the nearest context to the original sentence containing the ambiguous word.

To define this score measure we take in consideration the collocation similarity $\operatorname{Sim}\left(\mathrm{w}_{\mathrm{i}}, \mathrm{w}_{\mathrm{j}}\right)$ between the words $\mathrm{w}_{\mathrm{i}}$ contained in the original sentence $S$ and the words $\mathrm{w}_{\mathrm{j}}$ contained in the compared context of use CU.

Also the position from the ambiguous word (p) in the original sentence $\mathrm{p}$ will be considered in the score measure (see Equation 4).

$$
\left(\sum_{w_{i} \in S}\left(\sum_{w_{j} \in C U} \frac{1}{p} \times\left(\operatorname{Sim}\left(w_{i}, w_{j}\right)\right)\right) / m\right) / n
$$

Where $\mathrm{m}$ is the total number of words in the context of use and $\mathrm{n}$ is the total number of words in the sentence containing the word to be disambiguated. Those factors were used to calculate the average of similarity between the words contained in the sentence to be disambiguated and those contained in the context of use.
1) Measure of similarity between words $\operatorname{Sim}\left(w_{i}, w_{j}\right)$ : The collocation is an expression consisting of two or more words that correspond to some conventional way of saying things [10]. The similarity between words $\operatorname{Sim}(w i, w j)$ will be replaced by one of the collocation similarity defined in what follows.

a) The T-test: One of the most known metrics in the domain of the collocation retrieval, the t-test[10] is detailed in the equation 5 .

$$
\operatorname{Sim}\left(\mathrm{w}_{\mathrm{i}}, \mathrm{w}_{\mathrm{j}}\right)=\mathrm{T}=(\bar{x}-\mu) /\left(\sqrt{\frac{s^{2}}{N}}\right)
$$

The mean of the distribution $\mu$ is measured by multiplying $\mathrm{P}\left(\mathrm{w}_{\mathrm{i}}\right)$ to $\mathrm{P}\left(\mathrm{w}_{\mathrm{j}}\right)$, where $\mathrm{P}\left(\mathrm{w}_{\mathrm{i}}\right)=$ number of occurrences of $\mathrm{w}_{\mathrm{i}}$ in the corpus / Total number of words in the corpus. We notice that the sample variance $\mathrm{s}^{2}$ is equal to the sample mean $\bar{x}$, those factors are obtained by dividing the number of times where $\mathrm{w}_{\mathrm{i}}$ occurs with $\mathrm{w}_{\mathrm{j}}$ by $\mathrm{N}$ the total number of words in the corpus.

b) The Mutual Information. This measure determines how much a word can be informative for another word. The equation 6 in what follows details the mutual information measure [10].

$$
\operatorname{Sim}\left(\mathrm{w}_{\mathrm{i}}, \mathrm{w}_{\mathrm{j}}\right)=\operatorname{IM}\left(\mathrm{w}_{\mathrm{i}}, \mathrm{w}_{\mathrm{j}}\right)=\log _{2} \frac{P(w i, w j)}{P(w i) P(w j)}
$$

We notice that the probability of $\mathrm{w}_{\mathrm{i}}$ and $\mathrm{w}_{\mathrm{j}}$ are measured as follows:

$$
\begin{gathered}
\mathrm{P}\left(\mathrm{w}_{\mathrm{i}}, \mathrm{w}_{\mathrm{j}}\right)=\frac{\text { Number of times where } w_{i} \text { and } w_{j} \text { occurs together }}{\text { Total number of words in the corpus }} \\
\mathrm{P}(\mathrm{w})=\frac{\text { Number of occurences of } w}{N}
\end{gathered}
$$

This measure is dependent on the number of times where $\mathrm{w}_{\mathrm{i}}$ and $\mathrm{w}_{\mathrm{j}}$ occurs together. The collocations that occur more times together will have a lowest score than the collocations that don't have an important number of occurrences.

c) The Chi-Square. The $\chi 2$ test is not very different from the $\mathrm{T}$ test [10]. The basic principle is to count $\mathrm{C}_{1,1}$ (the number of occurrences of $\mathrm{w}_{\mathrm{i}}$ and $\mathrm{w}_{\mathrm{j}}$ ), $\mathrm{C}_{1,2}$ ( the number of occurrences of $\mathrm{w}_{\mathrm{i}}$ without $\mathrm{w}_{\mathrm{j}}$ ), $\mathrm{C}_{2,1}$ (the number of occurrence of $\mathrm{w}_{\mathrm{j}}$ without $\mathrm{w}_{\mathrm{i}}$ ) and $\mathrm{C}_{2,2}$ (the number of bigrams in the corpus that don't contains $\mathrm{w}_{\mathrm{i}}$ or $\mathrm{w}_{\mathrm{j}}$ ). The equation 8 in what follows details the measure of $\chi 2$.

$\operatorname{Sim}\left(\mathrm{w}_{\mathrm{i}}, \mathrm{w}_{\mathrm{j}}\right)=\frac{N \times\left(C_{1,1} \times C_{2,2}-C_{1,2} \times C_{2,1}\right)^{2}}{\left(C_{1,1}+C_{1,2}\right) \times\left(C_{1,1}+C_{2,1}\right) \times\left(C_{1,2}+C_{2,2}\right) \times\left(C_{2,1}+C_{2,2}\right)}$

For the different obtained results, we find that the highest score measure given by the $\mathrm{T}$ test are also given by the Chisquare.

2) The position from the ambiguous word ( $p)$ :The position of different words in the sentence from the ambiguous word is an important factor indicating the importance of the term. More the word is near to the ambiguous word, more the influence on the meaning of the word increases. 
For this, we assign a weight (p) for each word in the original sentence. This weight is affiliated according to the position of the corresponding word on the left or the right of the ambiguous word.

\section{The Vote Procedure}

The Vote procedure is a novel method that we define to choose the correct sense proposed by the different collocation measures defined in the previous section. The figure 2 below describes the architecture of the vote procedure.

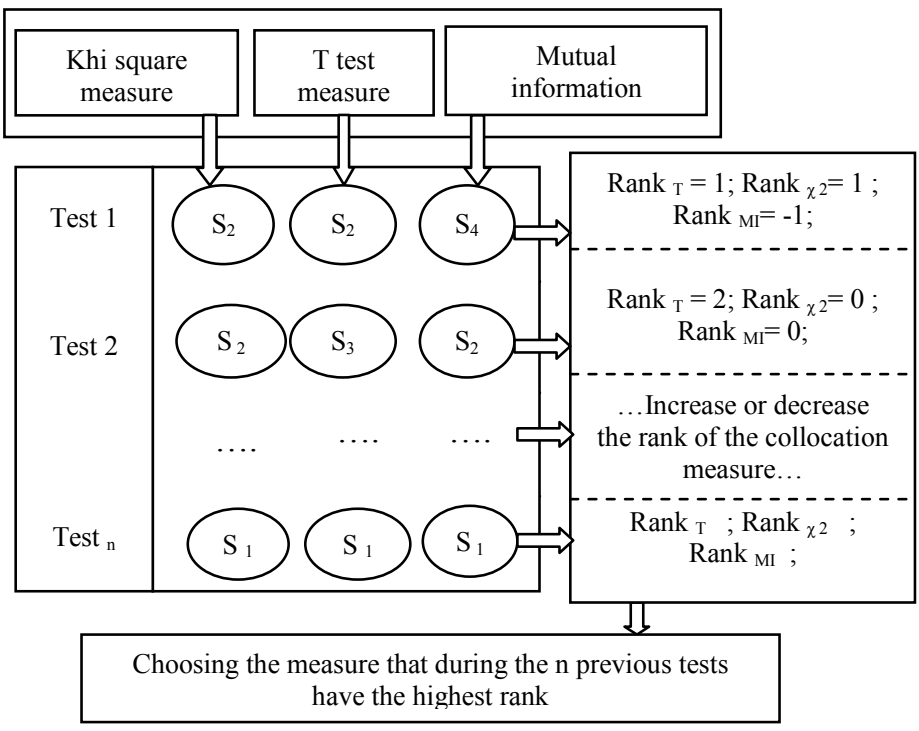

Figure2. Architecture of the vote procedure.

The idea is that during the experimental study, we ranked the measures of collocation according to the attribution of the sense.

In the case where the collocation measures agree on the same result, then the sense given by the measure will be attributed to the ambiguous word and the rank will not be changed.

The collocation measures can give different results, in the case where more than one measure agrees on the attribution of a sense to the ambiguous word, then, we have to choose the sense having the majority vote proposed by the measures. The rank of the collocation measures that vote for the attributed sense will be increased.

The final case is when all the collocation measures don't give the same result. Then, the result given by the collocation measure having the highest rank (attributed during the last $\mathrm{N}$ test) will be used to attribute the sense of the ambiguous word.

In the figure 2, the result of the $n$ tests is figured. During the first test (Test 1$)$, we find that the second sense $\left(\mathrm{S}_{2}\right)$ is given by the Chi-square and the $\mathrm{T}$ test. In contrast the fourth sense $\left(\mathrm{S}_{4}\right)$ was given by the mutual information. In this test, the rank of the Test $\left(\operatorname{Rank}_{\mathrm{T}}\right)$ and the Chi-square $\left(\operatorname{Rank}_{\chi_{2}}\right)$ is incremented, whereas the rank of the mutual information $\left(\operatorname{Rank}_{\mathrm{MI}}\right)$ is decreased. The same process is repeated for the other tests.

\section{EXPERIMENTAL RESULTS}

The English works were evaluated using Senseval, however in our work we have to make our experimental data using a totally different set of resources.

\section{A. Used tools and experimental data}

\section{1) Dictionary}

a) Description of the dictionary: To disambiguate Arabic words we need an Arabic-arabic dictionary that contains the different sense of the Arabic words, the problem in the traditional dictionary that some senses are not used in nowadays. We use the dictionary of "Lesan al arab" [11] that is one of the most known dictionary for the Arabic language.

b) Encountred Problems: During the experimental study we remark a difference between the number of senses given by a dictionary and those founded in the corpus. As a solution to this problem, we try to collect from the net some texts containing the missing senses and add them to our corpus.

For some considered words, we have found senses that appear in the corpus and don't exist in the dictionary. These senses were added to the list of candidate senses. For the word "ayn" we extract about ten sentences from the training corpus where it means a name of a city in United Arab Emirates. A sample is given in what follows: “ تستقبلنا مدينة العين ببهاء يختلف "The ayn city receives us brightly completely different than abu-dhabi”.

c) Sense Granularity: Most of the works cited in the literature, uses coarse grained senses, in this work we choose to work on fine-grained senses. In what follows, we can give the sample of the ambiguous word 《 أهل $»\langle$ ahl $»$, in the case where we work with coarse grained sense, we choose the sense family. In the case of fine grained sense, we use three senses cited in what follows:

- Sense1: 《 《he people of the house, its inhabitants »;

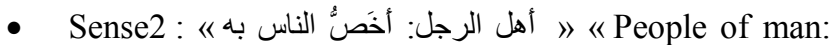
the people most concerned him »;

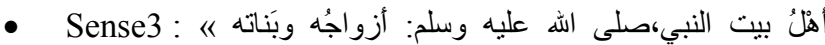

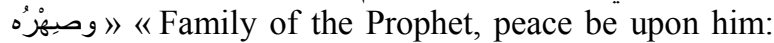
his wives and daughters and his brother $»$;

This choice makes our work more difficult and complex because it increases the number of the considered senses.

2) Corpus: We choose to work on texts dealing with multiple domains (sport, politics, religion, science, etc.).

These texts are extracted from newspaper articles, which were recorded from the different corpus that are available on the net. In total we collect a corpus that counts $123,854,642$ words.

\section{B. Obtained Results}

To measure the rate of disambiguation, we use the metric of the precision $\mathrm{P}$ (see Equation 10) and recall R (see Equation 11) [12].

$\mathrm{P}=$ Correct answers provided / Answers provided 


\section{$\mathrm{R}=$ Correct answers provided / Total answers to provide (11)}

Beginning with the tf_idf measure, used to remove stop words, according to an experimental study of Abu El-Khair [13], the tf_idf is the best weighting system for the Arabic language, without stemming. This is contradictory to other works where we find that BM25 works better for Arabic [14].

To justify the choice of the khoja stemmer for the stemming step, we compare the Al-shalabi Kanaan stemmer [15] to the Khoja stemmer. To do that, we test a sample of 935 words, contained in the glosses of the tested words, in the following table 1 , we details the number of errors and the rate of precision.

TABLEI. COMPARISON BETWEEN THE ALSHALABI KANAAN AND THE KHOJA STEMMER.

\begin{tabular}{|c|c|c|}
\hline $\begin{array}{c}\text { Stemming } \\
\text { algorithms }\end{array}$ & Alshalabi Kanaan stemmer & Khoja stemmer \\
\hline Errors & 231 & 356 \\
\hline Rate of precision & $0,76 \%$ & $0,63 \%$ \\
\hline
\end{tabular}

From the Table 1, we find that the Khoja stemmer is more performer than the Alshalabi kanaan stemmer. Since that we apply the approximate matching algorithm between the words belonging to the glosses and the paragraph containing the ambiguous word extracted from the corpus. As mentioned previously, the matching algorithm of words takes a long time during its execution. To facilitate this, we recorded occurrences of each root in our knowledge base. For some paragraph containing the ambiguous words, we haven't found any of the words contained in the glosses, as a solution, we affiliate the most frequent sense to them.

To determine the significant words, we select $\mathrm{k}$ words belonging to the obtained contexts of use, where $0<\mathrm{k} \leqslant \mathrm{n}-1$ ( $\mathrm{n}$ is the number of words in the context of use). In Table 2, we give the values of $\mathrm{k}$ for all contexts of use corresponding to the different senses of some ambiguous words. We give also the number of words in different contexts of use corresponding to different meanings and the total number of contexts of use.

TABLEII. STATISTICS OF THE CONTEXTS OF USE FOR SOME AMBIGUOUS WORDS.

\begin{tabular}{|c|c|c|c|c|}
\hline $\begin{array}{c}\text { Ambiguou } \\
\text { S words }\end{array}$ & $\begin{array}{c}\text { Transcr } \\
\text { iption }\end{array}$ & $\begin{array}{c}\text { Total } \\
\text { number of } \\
\text { use contexts }\end{array}$ & $\begin{array}{c}\text { Total } \\
\text { number of } \\
\text { words }\end{array}$ & $\begin{array}{c}\text { K : } \\
\text { Number of } \\
\text { significant } \\
\text { words }\end{array}$ \\
\hline عقين & ayn & 44,543 & 743,442 & 383,329 \\
\hline عقر & chaar & 28,733 & 489,214 & 139,077 \\
\hline Far & akl & 10,544 & 179,375 & 50,139 \\
\hline
\end{tabular}

As we have already noted that there is a difference between the number of senses defined in the dictionary and the senses founded in the corpus. On the other hand the number of contexts of use found in the corpus varies from one sense to another one for each ambiguous word. We give in figure 3, the percentage of contexts of use for each sense of the ambiguous word " شعر "' chaar ". This percentage is obtained by dividing the number of contexts of use affiliated to the sense Si by the total number of contexts of use.

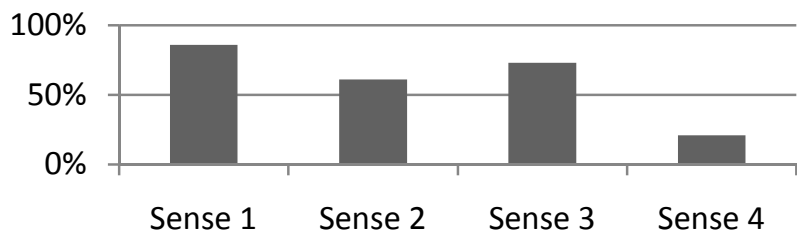

Figure 3. Percentage of the contexts for each sense of the ambiguous word « شعر 》 « chaar».

The variation in number of contexts between the different senses of the ambiguous word affect the obtained results, since it reduces the accuracy rate for the tested sentences that contains a rarely sense. To test the effectiveness of our system, we test 20 sentences for each sense founded in the corpus.

The rate of precision varies between the different senses of the word, while the number of tested sentence is the same. This shows that the decrease of the accuracy rate is due to the reduced number of contexts of use. Taking the case of 4 Sense of ambiguous word "شعر" "chaar", the lowest percentage of contexts of use corresponds to the lowest accuracy rate.

We conclude that the decrease in the rate of precision is mainly due to the insufficient number of contexts of use, which leads the failure to respond to all possible events. For that we try to collect as many texts as we can, to expand the number of contexts of use.

We notice that the collocation measures give different results for the same tested ambiguous word. The use of the vote procedure is necessary in our work. The accuracy rate of our system varies from one to another ambiguous word.

In table 3 below we give the rate of Precision and Recall for fifty words.

TABLEIII. OBTAINED RESULTS GIVEN BY THE PROPOSED METHOD.

\begin{tabular}{|c|c|c|}
\hline & Final rate & MFS \\
\hline Precision & 0,79 & 0,87 \\
\hline Recall & 0,68 & 0,75 \\
\hline
\end{tabular}

The proposed method gives satisfactory results in the average of $79 \%$ and $87 \%$ for the most frequent sense. A comparison of the obtained results by other systems using similar ambiguous words, sentences and resources that we used in our work, seems impossible. For that, in what follows, we detail the results given by some works of WSD that was experimented using the same data and resources.

\section{Comparison between our work and related works}

The words evaluated with the variants of the Lesk algorithm and the test samples, are used in the experimental study of this work. Most works that has been achieved for WSD of the other languages were evaluated by SemEval. These works were tested using the same resources, the same samples and the same granularity of ambiguous words.

In contrast the works that was described previously in section 2 are experimented using different data and resources. 
Table 4 contains the accuracy obtained by the different works, and the number of tested words.

TABLE IV. COMPARISON BETWEEN DIFFERENT WORKS OF ARABIC WSD.

\begin{tabular}{|c|c|c|c|}
\hline \multicolumn{2}{|l|}{ Systems } & $\begin{array}{l}\text { Number of } \\
\text { tested words }\end{array}$ & $\begin{array}{l}\text { Rate of } \\
\text { precision }\end{array}$ \\
\hline \multicolumn{2}{|l|}{$S A L A A M$} & 1071 & $56,9 \%$ \\
\hline \multicolumn{2}{|c|}{ Nä̈ve Bayesian classifier } & 4 & $76,66 \%$ \\
\hline \multicolumn{2}{|c|}{$\begin{array}{l}\text { Combination of information retrieval } \\
\text { measures with the Lesk algorithm }\end{array}$} & 10 & $76 \%$ \\
\hline \multicolumn{2}{|c|}{ Original Lesk Algorithm } & 50 & $59 \%$ \\
\hline \multirow{5}{*}{$\begin{array}{l}\text { Modified } \\
\text { Lesk } \\
\text { Algorithm }\end{array}$} & $\begin{array}{l}\text { Wu and Palmer } \\
\text { (ComSim) }\end{array}$ & 50 & $56 \%$ \\
\hline & Resnik (SimRes) & 50 & $33 \%$ \\
\hline & $\begin{array}{l}\text { Jiang and Conrath } \\
\text { (RelJcn) }\end{array}$ & 50 & $33 \%$ \\
\hline & Lin(RelLin) & 50 & $44 \%$ \\
\hline & $\begin{array}{l}\text { Leacock and Chodorow } \\
\text { (SimLch) }\end{array}$ & 50 & $67 \%$ \\
\hline \multicolumn{2}{|c|}{ Our Method } & 50 & $79 \%$ \\
\hline
\end{tabular}

The fact that the naive Bayesian classifier gets a higher accuracy rate does not mean that he is more efficient than SALAAM system given the large number of data tested.

The modified version of the Lesk algorithm obtains an accuracy rate of $67 \%$. The best accuracy rate is obtained for a window size of three words using the simplified algorithm Lesk, and two words by using the modified algorithm of Lesk (based on the use of AWN). Among these proposals, Leacock and Chodorow (SimLch) measure performs best rate of precision.

Compared to our method, we note that the Lesk algorithm is very expensive because of the enormous amount of data. This algorithm is also limited to dictionary definitions that we use therefore the absence of a certain word can radically change the results. On the other hand, for the modified version of the Lesk algorithm, we find that AWN has an insufficient coverage. Indeed by comparing for example the number of glosses in the dictionary considered in relation to those of AWN for fifty words tested, we find that AWN has an average coverage rate of about $63.63 \%$.

In addition the effect of the position of the word in the sentence is not taken into account in the score. The words closest to the ambiguous word generally have an influence on the result of disambiguation. Finally and comparing the proposed method to the combination of information retrieval measures with the Lesk algorithm, we increase the rate of precision by $3 \%$ using more ambiguous words during the experimental study.

\section{CONCLUSION}

This paper has presented an unsupervised method to disambiguate Arabic words. A context of use will be generated following some steps (elimination of stop words, stemming and the approximate string matching algorithm) for each sense of the ambiguous word.

We measure the similarity between the contexts of use corresponding to the glosses of the word to be disambiguated and the original sentence. The similarity measure affiliates a score using three collocation measures that will be ranked by the vote procedure. The sense proposed by the most ranked score, is chosen as the correct sense of the ambiguous word.

We propose that in future works to integrate some other collocation measures to perform our system.

\section{REFERENCES}

[1] E. Agirre and P. Edmond, "Word Sense Disambiguation: Algorithms and Applications", published in Springer (www.wsdbook.org), 2006.

[2] S. Khoja, R. Garside, "Stemming Arabic text", www.comp.lancs.ac.uk/computing/users /khoja/stemmer.ps, 1999.

[3] M. Elloumi, "Comparison of Strings Belonging to the Same Family", Information Sciences, An International Journal, Vol. 111, Issue 1-4, Elsevier Publishing Co., Amsterdam, North-Holland (Publisher), 1998, p.p. 49-63.

[4] Y. Guiassa et F. M. Hayet, "Désambiguïsation lexicale d'un texte arabe", Conférence TALN'06 (Traitement automatique des langues naturelles), Leuven, Belgique, 2006.

[5] A. Zouaghi, L. Merhbene, M. Zrigui, "Word Sense disambiguation for Arabic language using the variants of the Lesk algorithm", in Proceeding of the International Conference on Artificial Intelligence (ICAI'11),USA: Las Vegas, 2011, p.p. 561-567.

[6] A. Zouaghi, L. Merhbene, M. Zrigui, "Combination of information retrieval methods with LESK algorithm for Arabic word sense disambiguation". Journal Article published in the Artificial Intelligence Review, Online First, 30 May 2011; DOI: 10.1007/s10462-011-9249-3; Online ISSN: 1573-7462.

[7] M. Diab, P. Resnik, "An unsupervised method for word sense tagging using parallel corpora", in Proc. 40th Meeting of the Association for Computational Linguistics, Philadelphia, U.S.A, 2002, pp. 255-262.

[8] S. Elmougy, H. Taher and H. Noaman, "Naïve Bayes Classifier for Arabic Word Sense Disambiguation", in proceeding of the Informatics and Systems (INFOS2008), Cairo-Egypt, March 27-29, 2008.

[9] G. Salton and C. Buckley: Term-weighting approaches in automatic text retrieval. Information Processing and Management, 24 (5), pp. 513-523., 1998.

[10] H. Schutze and C. Manning "Foundations of Statistical Natural Language Processing". MIT Press, Cambridge, MA, 1999, ISBN 978-0262-13360-9.

[11] M. Ben Mukarram al-Ifriqi and M.al-Misri, "Lisàn al-'arab", édition libanaise imprimée du Lisàn al-'arab -- Ibn Manzûr, Lisàn al-'arab, 15 volumes. Beyrouth, 1956.

[12] R. Navigili, "Word Sense Disambiguation : A Survey", ACM Computing Surveys, Vol. 41, No. 2, Article 10, 2009.

[13] Abu El-Khair, "Effects of stop words Elimination for Arabic information retrieval: A comparative study", Published in the International Journal of Computing \& Information Sciences. Vol4. No 3, 2006.

[14] J. Savoy, Rasolofo, "repost on the TREC-11 Experiment : Arabic, Name Page and Topic Distillation Searches", published in the proceeding of the Eleventh Text Retrival Conference, TREC, 2002.

[15] R. Al-Shalabi, G. Kanaan, Al-Serhan, H, "New approach for extracting Arabic roots", in Proc. International Arab Conference on Information Technology, (ACIT'2003), , Egypt, 2003, p. 307. 